\title{
LA EFECTIVIDAD DEL ALBENDAZOL EN EL TRATAMIENTO DE LA ESTRONGILOIDIASIS EN NIÑOS
}

\author{
A. DOMINGUEZ*, A. ALZATE**
}

\begin{abstract}
En la localidad de Córdoba, Municipio de Buenaventura, Colombia, se detectó una prevalencia de $S$. stercoralis del $16 \%$ en menores de seis años. En un grupo de 13 niños se evaluó la eficacia y tolerancia de un nuevo agente antihelmíntico, el Albendazol. Los niños de ambos sexos ( $6 \mathrm{M} / 7 \mathrm{~F}$ ) con rango de edad de dos a seis años, recibieron $400 \mathrm{mg}$ diariamente en una sola dosis oral, durante tres días consecutivos.
\end{abstract}

La tasa de curación fue estimada, utilizando la técnica de Baermann, con muestras obtenidas a los 7 y 21 días después de la dosis final del tratamiento. El porcentaje de curación de $76.9 \%$, encontrándose una excelente tolerancia a la droga. Por lo tanto, se podría considerar al Albendazol como la droga de elección en este tipo de parasitosis.

\section{INTRODUCCION}

La infección por $S$. stercoralis es en ocasiones asintomática, teniendo una prevalencia en América Latina alrededor del $10 \%$ o menos y en Colombia únicamente del 2\%, según reveló la Encuesta Nacional de Salud (1), en donde se utilizó como diagnóstico el método directo, el cual no es el ideal para detectar esta parasitosis humana, por lo que podría considerarse que la prevalencia detectada en ese estudio sea en realidad más alta.

En la actualidad se cuenta con drogas altamente efectivas para parasitosis intestinales; sin embargo, algunos de estos fármacos tienen espectro muy reducido, con efectos colaterales importantes y por lo tanto poca aplicación en tratamientos masivos. En cambio los fármacos antiparasitarios con amplio espectro, administración en dosis únicas y buena tolerancia, facilitan indudablemente los tratamientos a nivel comunitario.

Entre las drogas contra helmintos transmitidos por el suelo figuran el Pamoato de pirantel, el Pamoato de oxantel, la Piperazina, el grupo de los benzimidazoles, tales como el Mebendazol, Tiabendazol, Levamisol y más recientemente al Albendazol (2).

El Albendazol tiene ciertas ventajas ante los otros fármacos del grupo de los benzimidazoles, específicamente contra $A$. lumbricoides, $T$. trichiura, $N$. americanus y $A$. duodenale $(3,4,5)$. Sin embargo, la eficacia contra $S$. stercoralis es incierta debido a la discordancia de los resultados comunicados en diversas publicaciones, las cuales van del $28 \%$ de curaciones (6), al $88 \%(4,5)$.

El Tiabendazol, medicamento con rápida y potente absorción, tiene eficacia contra los nemátodos tisulares y es el fármaco más utilizado en el tratamiento de la estrongiloidiasis. Con una tasa de curación del 75 al 95\% (7), tiene la desventaja de presentar efectos colaterales importantes que restringen su aplicación masiva (8).

Los estudios realizados tanto en animales como en humanos han demostrado que el Albendazol posee actividad vermicida, ovicida y larvicida (9), en dosis

\footnotetext{
* Estudiante de posgrado, Universidad del Valle, Cali, Colombia. Investigador del Centro de Investigaciones Ecológicas del Sureste. CIES, Carretera Panamericana Periférico Sur s/n., San Cristobal de Las Casas Chiapas, México, 29290.

** Jefe del Departamento de Microbiología, Universidad del Valle, Cali, Colombia.
} 
de $400 \mathrm{mg}$. Se considera que la droga ejerce su efecto antihelmínico bloqueando la captación de glucosa por los parásitos susceptibles. Principalmente la acción antihelmítica del Albendazol es intra-intestinal, teniendo una baja absorción después de la administración oral, alcanzando su principal metabolito una vida media de 8.5 horas en el plasma, el cual se elimina principalmente por la orina.

En un estudio realizado en la localidad de Córdoba, Buenaventura, Colombia, donde se estaban estudiando factores de riesgo para la malaria en niños, se efectuaron, entre otros, exámenes coprológicos (10). La elevada prevalencia de $S$. stercoralis en esta localidad presentaba la oportunidad de evaluar una droga alterna para el tratamiento de la infección, sobre todo considerando que el Tiabendazol, ha sido descontinuado en Colombia y el cual se venía utilizando en el tratamiento de esta parasitosis.

Por lo antes señalado, se llevó a cabo un estudio con el fin de evaluar la efectividad y toxicidad del Albendazol en niños infectados con $\boldsymbol{S}$. stercoralis.

\section{MATERIAL Y METODOS}

El estudio se realizó en la comunidad de Córdoba, del Municipio de Buenaventura, en la costa Pacífica colombiana; ubicada a $20 \mathrm{~km}$. de la cabecera municipal, con una altitud de $25^{\circ} \mathrm{C}$, con pluviosidad anual superior a $8000 \mathrm{~mm}$ y con una humedad relativa de $88 \%$. Aproximadamente con 573 habitantes, predominando la raza negra.

Se examinaron a 127 niños menores de 6 años de edad en busca de helmintos intestinales. El examen cropológico fue hecho con el método directo, detectándose el $16 \%$ de infección por $S$. stercoralis. Se estudiaron 18 niños de ambos sexos, entre 2 y 6 años de edad por medio de la técnica de Baermann (12) antes del tratamiento. Se trataron luego con $400 \mathrm{mg}$ de Albendazol (metil 5-propiltio-1- benzimidazol-2-il-carbamato) en una dosis única, cada 24 horas, durante tres días consecutivos.

Para demostrar la actividad antihelmíntica del fármaco, se repitieron con la misma técnica los exámenes de heces a los siete y 21 días después del tratamiento; estableciéndose que serían considerados curados aquellos niños con coproparasitoscópico negativo, en los días señalados, para los exámenes de control.
El tratamiento fue administrado por uno de los investigadores y se vigiló estrechamente a los niños, recolectándose información acerca de los efectos colaterales indeseables, atribuibles al Albendazol.

\section{RESULTADOS}

De los 18 niños seleccionados para el estudio, únicamente 13 de ellos lo completaron. Los hallazgos se muestran en el Cuadro 1, donde observamos que la tasa de curación fue del $76.9 \%$.

De los 13 niños que participaron en el estudio de $46 \%$ fueron del sexo masculino y el $54 \%$ del femenino; el rango de edad osciló entre dos y seis años de edad, con un promedio de 4.0 y una desviación de 1.1 años.

En relación a los efectos colaterales, éstos no se observaron en ninguno de los niños tratados, lo cual habla de una baja toxicidad de la droga.

\section{DISCUSION}

Al revisar la bibliografía en relación a la efectividad del Albendazol sobre $S$. stercoralis $(4,5,6)$, los hallazgos son un tanto contradictorios, ya que la mayoría de los estudios manejan un número escaso de pacientes, dada la dificultad de los métodos de detección y la heterogeneidad en edad de los pacientes estudiados.

CUADRO 1

RESULTADOS DEL TRATAMIENTO DE ESTRONGILOIDIASIS POR MEDIO DEL ALBENDAZOL ( $400 \mathrm{mg}$ EN DOSIS UNICA DIARIA, DURANTE 3 DIAS), EN I MENORES DE 6 AÑOS

\begin{tabular}{|c|c|c|c|}
\hline $\begin{array}{c}\text { C A so } \\
\text { n. }\end{array}$ & $\begin{array}{l}\text { EOAD } \\
\text { EIf } \\
\text { Ailos }\end{array}$ & $S E \times 0$ & RESULTADO \\
\hline 1 & 4 & F & $c$ \\
\hline 2 & 3 & n & $c$ \\
\hline 3 & 5 & $\mathbf{F}$ & $c$ \\
\hline 4 & 4 & $F$ & 0 \\
\hline 8 & 2 & $F$ & $c$ \\
\hline 1 & 2 & n & II \\
\hline 1 & 4 & $\mathbf{F}$ & $c$ \\
\hline 1 & 3 & n & $c$ \\
\hline 9 & 3 & " & 110 \\
\hline 10 & 4 & F & $c$ \\
\hline 11 & 5 & " & c \\
\hline 12 & 4 & $\mathbf{F}$ & c \\
\hline 13 & 4 & " & Mo \\
\hline
\end{tabular}


En este estudio el Albendazol resultó con una tasa de curación del $76,9 \%$ a los 21 días después del tratamiento, la cual es muy similar a la efectividad que se obtienen con el Tiabendazol, cuyo porcentaje de curación es de 75-95\% (7), estando ya descontinuado en Colombia y empleado para el tratamiento de la estrongiloidiasis, con la desventaja de presentar efectos colaterales importantes (8). Nuestros resultados con Albendazol son comparables con los obtenidos por otros investigadores $(4,5)$, sin embargo, es importante señalar que una característica biológica de este parásito, es la de evaluar la eficacia de drogas, recurrir a métodos coproparasitoscópicos apropiados en serie de tres y si es posible alternadamente $(11,12)$.

A pesar de que el diseño experimental en este estudio no fue el adecuado, los resultados obtenidos son alentadores para continuar investigando más sobre este particular.

\section{SUMMARY}

The efficacy and tolerance of a new anthelmintic drug, Albendazole, was determined in the tratment of 13 children infected with $S$. stercoralis, in Córdoba, municipality of Buenaventura, Colombia. The study comprising children aged 2-6 years, of both sexes, includet administration of $400 \mathrm{mg}$ daily as a single oral dose, for three consecutive days.

From a initial prevalence of $16 \%(n=124)$, the cure rates were assessed following Baermann's technique, with samples obtained 7 and 21 days; after the final dose of the drug. A mean cure rate of $76.9 \%$ was achieved, with an excellent tolerance to the drug. From this results, it may be suggested that Albendazole should be considered the drug of choice for this of parasite.

\section{AGRADECIMIENTOS}

Expresamos nuestro sincero reconocimiento a la compañía Smith Kline and French de Colombia, por haber proporcionado la droga (ALBENDAZOL) y a la Bióloga Fanny de Carvajal, del Departamento de Microbiología de la Universidad del Valle, por haber practicado los exámenes coprológicos.
Esta investigación recibió apoyo financiero del Programa Especial para Investigación y Entrenamiento en Enfermedades Tropicales PNUD/Banco Mundial/ WHO.

\section{BIBLIOGRAFIA}

1. Ministerio de Salud Pública y Asociación Colombiana de Facultades de Medicina, Bogotá, Colombia. Investigación Nacional de Morbilidad. Parasitismo Intestinal, 1969; 62.

2. Botero D. Guías para el tratamiento de las parasitosis intestinales frecuentes en Colombia. Biomédica, 1983; 3: 130.

3. Amato Neto V, Baillot Moreira AA, Campos R, y cols. Tratamiento de ancilostomiase, ascaridiase e tricocefaliase por medio do Albendazol ou do Mebendazol. Rev Inst Med Trop Sao Paulo, 1983; 25: 294.

4. Pene P, Mojon M, Garin JP, Coulaud JP, Rossignol JF. Albendazole: A new broad spectrum anthelmintic Doubleblind multicenter clinical trial. Am J Trop Med.Hyg, 1982; 31: 263 .

5. Rossignol JF, Maisonneuve H. Albendazole: Placebocontrolled study in 870 patients with intestinal helminthiasis. Trans R Soc Trop Med Hyg, 1983; 77: 707.

6. Amato Neto V, Baillot Moreira AA, Gianizella Chiamarelli MC, y Col. Demarcacao da actividade anti-helmintica do Albendazol. Estudo referente a estrongiloidiase humana. Rev Inst Med Trop Sao Paulo, 1985; 27: 95.

7. Yokogawa M. Antihelmínicos actuales en el tratamiento masivo de las helmintiasis transmitidas por el suelo. Salud Pública Mex, 1985; 27: 149.

8. Botero D. Epidemiology and Public Health Importance of Intestinal Nematode Infecions in Latin America. En: Progress in Drug Research Birkhauser Verlag, Basel und Stuttgart, 1975; 19: 20.

9. Cline BL, Little MD, Bartholomew RK, Halsey NA. Larvicidal activity of Albendazol against Necator americanus in human volunteers. Am J Trop Med Hyg, 1984; 33: 387.

10. Domínguez A. Características sociodemográficas relacionadas con la malaria en menores de seis años en la costa del pacífico colombiano. Tesis. Magisters en Ciencias en Epidemiología, 1986, 163 pp. Universidad del Valle, Cali, Colombia.

11. Botero D. Clinical trial methodology in intestinal parasitic diseases. Clin Pharmacol Ther, 1976; 19: 630.

12. Lumbreras H. Strongyloidiosis: 1 . Evaluación de la "técnica de Baermann modificada en copa" en el estudio de la estrongyloidiosis Rev Médica Peruana, 1963; 32: 119. 